\title{
SOLUBLE GROUPS IN WHICH EVERY FINITELY GENERATED SUBGROUP IS FINITELY PRESENTED
}

\author{
J. R. J. GROVES \\ (Received 10 December 1976; revised 7 September 1977) \\ Communicated by M. F. Newman
}

\begin{abstract}
The class of finitely generated soluble coherent groups is considered. It is shown that these of ways. In particular, they are precisely the class of finitely generated soluble groups $G$ with the property:

$$
\left|\begin{array}{l}
\text { if } x, y \in G \text { then either the subgroup generated by } \\
\left\{y^{-i} x y^{i}: i \geqslant 0\right\} \text { or the subgroup generated by } \\
\left\{y^{-i} x y^{i}: i \leqslant 0\right\} \text { is finitely generated. }
\end{array}\right|
$$
\end{abstract}
groups have the maximal condition on normal subgroups and can be characterized in a number

Subject classification (Amer. Math. Soc. (MOS) 1970): primary 20 E 15; secondary 20 F 05.

\section{Introduction}

The question of which soluble groups are finitely presented has been receiving some attention in recent years (see Baumslag, 1974). The main tenor of the results obtained appears to be that the property of being finitely presented imposes very little structure on a soluble group. For example, Baumslag (1973) has shown that every finitely generated metabelian group can be embedded in a finitely presented metabelian group.

In this paper, we approach a stronger condition than that of being finitely presented. We call a group coherent if all of its finitely generated subgroups are finitely presented and we show that coherence places a considerable restriction on the structure of finitely generated soluble groups. To explain this, we require some 
notation. Call a group G semi-polycyclic if it is finitely generated, soluble and satisfies the following condition:

for all $x, y \in G$, at least one of the subgroups generated by $\left\{x^{y^{i}}: i \geqslant 0\right\}$ and $\left\{x^{u^{i}}: i \leqslant 0\right\}$ is finitely generated.

(The notation derives from the fact that requiring both subgroups to be finitely generated is equivalent to polycyclicity.)

THEOREM. Let $G$ be a finitely generated soluble group. Then $G$ is coherent if and only if it is semi-polycyclic.

The proof that semi-polycyclic groups are coherent proceeds by showing that they have a well-defined structure intermediate between that of polycyclic groups and that of soluble linear groups. This structure is then used to show that they are finitely presented.

The converse is proved by first using a theorem of Bieri and Strebel (1977) to show that finitely generated coherent soluble groups have the maximum condition on normal subgroups. Hence each of the finitely generated sections in such a group is finitely presented. Now define the group $M(\alpha, p)$, where $\alpha$ is an element of some field of characteristic $p$, to be the group generated by the matrices

$$
\left\{\left[\begin{array}{ll}
1 & 0 \\
1 & 1
\end{array}\right]\left[\begin{array}{ll}
\alpha & 0 \\
0 & 1
\end{array}\right]\right\} .
$$

Then it is not difficult to show that $M(\alpha, p)$ is finitely presented if and only if

(i) $p \neq 0$ and $\alpha$ is algebraic over the prime field, or

(ii) $p=0$ and one of $\alpha$ or $\alpha^{-1}$ is an algebraic integer.

The proof of the theorem is completed by showing that each finitely generated soluble group which is not semi-polycyclic contains a section isomorphic to some non-finitely presented $M(\alpha, p)$.

Finally, it is of interest to note that, using the structure obtained for semipolycyclic groups and their equivalence with coherent groups, we can characterize either class described in the theorem as the class of groups which are HNNextensions having polycyclic base group and an associated subgroup equal to the base group. That is, the class of groups with a presentation $\left\langle V, t: V \varphi=V^{t}\right\rangle$, where $V$ is polycyclic and $\varphi: V \rightarrow V$ is a monomorphism (compare with Bieri and Strebel, 1977).

\section{Notation and preliminary results}

We refer to the book of Robinson (1972) for unexplained notation and basic results on soluble groups. We shall use $J, J_{+}$and $J_{-}$to denote, respectively, the 
sets of integers, of non-negative integers and of non-positive integers. We use special symbols for the following classes of groups:
$\mathfrak{U}$ abelian
(5) finitely generated
$\mathfrak{N}$ nilpotent
$Q$ polycyclic
F finite soluble
$Q_{1}$ semi-polycyclic

Suppose that $G$ is a group, $H$ a normal subgroup of $G$ and $g \in G$. Then we say that $g$ has

(a) nilpotent, (b) polycyclic, (c) semi-polycyclic, action on $H$ if, for each $h \in H$,

(a) $g$ is an Engel element in $\langle g, h\rangle$,

(b) $\left\langle h^{\alpha^{d}}: i \in J\right\rangle$ is finitely generated,

(c) either $\left\langle h^{o^{i}}: i \in J_{+}\right\rangle$or $\left\langle h^{o^{i}}: i \in J_{-}\right\rangle$is finitely generated.

We shall also denote by $\mathfrak{I}$ the class of finitely generated soluble groups $G$ having a normal series

satisfying

$$
\{1\} \leqslant N \leqslant C \leqslant H \leqslant G
$$

(a) $G / H$ is finite, $\quad$ (b) $H / C$ is cyclic,

(c) $H / N$ is abelian, (d) $N$ is nilpotent,

(e) each element of $C$ has polycyclic action on $N$ and each element of $H$ has semi-polycyclic action on $C$.

A series of this type will be called a $\mathfrak{I}$ series. (The class of groups $\mathfrak{I}$ will be shown, in the next section, to be precisely the class of semi-polycyclic groups.)

We begin our proofs with some elementary observations about the classes $\mathfrak{Q}_{1}$ and $\mathfrak{I}$.

LEMMA 1. If $\mathfrak{X}=\mathfrak{Q}_{1}$ or $\mathfrak{X}=\mathfrak{I}$ then $S \mathfrak{X} \cap \mathfrak{G}=Q \mathfrak{X}=\mathfrak{F} \mathfrak{X}=\mathfrak{X} \mathfrak{F}=\mathfrak{X}$.

PROOF. The proofs are largely straightforward-the only thing likely to give trouble being the proof that $\mathfrak{Q}_{1} \mathfrak{F}=\mathfrak{Q}_{1}$. By induction, we can reduce this to the case where $H$ is a normal subgroup of a finitely generated soluble group $G$ with $G / H$ finite and abelian. If $g, h \in G$, observe that $[g, h], g^{n} \in H$, for some $n$ and so $\left\langle[g, h]^{\boldsymbol{g}^{n i}}: i \in J_{+}\right\rangle$(say) is finitely generated. It follows easily that $\left\langle[g, h]^{\boldsymbol{a}^{i}}: i \in J_{+}\right\rangle$, and so $\left\langle h^{\sigma^{*}}: i \in J_{+}\right\rangle$, is finitely generated.

LeMmA 2. Let $G$ be a soluble group, $H$ a normal subgroup of $G, x \in G$, and suppose that $G=\langle H, x\rangle$.

(a) If $H \in L Q$ and $x$ has polycyclic action on $H$, then $G \in L Q$.

(b) If $H \in L Q$ and $x$ has semi-polycyclic action on $H$, then (i) there exists $\varepsilon= \pm 1$ such that, for all $h \in H,\left\langle h^{x^{e t}}: i \in J_{+}\right\rangle$is finitely generated and (ii) if $G$ is also finitely generated, $G \in \mathfrak{Q}_{1}$. 
Proof. (a) Suppose that $h_{1} x^{\alpha_{1}} \ldots h_{n} x^{\alpha_{n}} \in G$, with $h_{i} \in H, \alpha_{i} \in J$. Since $x$ has polycyclic action on $H$, the group $K=\left\langle h_{i}^{x^{j}}: j \in J, i=1, \ldots, n\right\rangle$ is finitely generated and so, because $H \in L Q$, is polycyclic. But $x$ normalizes $K$ and so $\langle K, x\rangle$ is also polycyclic. Since $\left\langle h_{1} x^{\alpha_{1}}, \ldots, h_{n} x^{\alpha_{n}}\right\rangle \leqslant\langle K, x\rangle$, the former subgroup is also polycyclic, showing that $G \in L \mathbb{Q}$.

(b) (i) Suppose there exist $h_{1}, h_{2} \in H$ such that $\left\langle h_{1}^{x^{i}}: i \in J_{+}\right\rangle$and $\left\langle h_{2}^{x^{i}}: i \in J_{-}\right\rangle$are both not finitely generated. Since $x$ has semi-polycyclic action on $H$, either $\left\langle\left(h_{1} h_{2}\right)^{x^{i}}: i \in J_{+}\right\rangle$or $\left\langle\left(h_{1} h_{2}\right)^{x^{i}}: i \in J_{-}\right\rangle$is finitely generated-suppose the former. Then $\left\langle h_{2}^{x^{i}}: i \in J_{+}\right\rangle$is finitely generated and so $\left\langle\left(h_{1} h_{2}\right)^{x^{i}}, h_{2}^{x^{i}}: i \in J_{+}\right\rangle$is polycyclic. But $\left\langle h_{\mathbf{1}}^{x^{i}}: i \in J_{+}\right\rangle$is a subgroup of this and so is also finitely generated. This contradiction to our initial assumption proves the result.

(b) (ii) Suppose, by part (i), that $\left\langle h^{x^{i}}: i \in J_{+}\right\rangle$is finitely generated for all $h \in H$. Let $h_{1} x^{\alpha}, h_{2} x^{\beta} \in G$ with $h_{1}, h_{2} \in H, \alpha, \beta \in J$; let $g_{1}=h_{1} x^{\alpha}$ and let $g_{2}$ be $h_{2} x^{\beta}$ if $\beta \geqslant 0$ or $\left(h_{2} x^{\beta}\right)^{-1}$ if $\beta<0$ (we assume, for convenience, that $\beta \geqslant 0$ ). Since

$$
\left\langle g_{1}^{g^{i}}: i \in J_{+}\right\rangle=\left\langle g_{1},\left[g_{1}, g_{2}^{i}\right]: i \in J_{+}\right\rangle,
$$

it suffices to show that $\left\langle\left[g_{1}, g_{2}^{i}\right]: i \in J_{+}\right\rangle$is finitely generated.

Now $g_{2}^{i}=\left(h_{2} x^{\beta}\right)^{i}=h_{2} x^{\beta i}$ where $h_{2} \in\left\langle h_{2}^{x^{\beta i}}: i \in J_{+}\right\rangle$and so

$$
\left[g_{1}, g_{2}^{i}\right]=\left[h_{1} x^{\alpha}, h_{2} x^{\beta i}\right]=\left[h_{1}, x^{\beta i}\right]^{x^{\alpha}}\left[h_{1}, h_{2}\right]^{x^{\beta i+\alpha}}\left[x^{\alpha}, h_{2}\right]^{x^{\beta i}} \text {. }
$$

Thus $\left\langle\left[g_{1}, g_{2}^{i}\right]: i \in J_{+}\right\rangle \leqslant\left\langle h_{1}^{x^{i}}, h_{2}^{x^{i}}: i \in J_{+}\right\rangle$. Since $x$ has semi-polycyclic action on $H$, the latter group is finitely generated; since, also, $H \in L Q$, the former group is also finitely generated. Thus $G \in \mathfrak{Q}_{1}$, as required.

Proposition 3. $\mathfrak{I} \leqslant \mathfrak{Q}_{1}$.

Proof. Let $G \in \mathfrak{I}$ and let

$$
\{1\} \leqslant N \leqslant C \leqslant H \leqslant G
$$

be a $\mathfrak{Z}$-series for $G$. Since $N$ is nilpotent it is locally polycyclic. Since $C$ has polycyclic action on $N$ and $C / N$ is abelian, $C$ is locally polycyclic, by (i) of Lemma 2 . Since $H$ has polycyclic action on $C$ and $H / C$ is cyclic, $H$ is semi-polycyclic, by (ii) (b) of Lemma 2. Finally, $G$ is semi-polycyclic by Lemma 1.

We complete this section with two technical lemmas which will be used in the next section.

LEMma 4. Let $G \in \mathfrak{T}$, let

$$
\{1\} \leqslant N \leqslant C \leqslant H \leqslant G
$$

be a I-series for $G$ and suppose that $G=H=\langle C, x\rangle$. Suppose that $G$ has max-n. 
Then $N$ has a finite central series

$$
\{1\}=N_{0} \leqslant N_{1} \leqslant \ldots \leqslant N_{k}=N
$$

such that $N_{i+1}=\left\langle N_{i}, d_{i+1}^{x^{j}}: j \in J\right\rangle$ for some $d_{i+1} \in N_{i+1}$.

ProOf. By induction on the class of $N$, we can suppose that $N$ has a finite central series

$$
\{1\}=N_{0}<N_{1}<\ldots<N_{k}=N
$$

with $N_{i+1} / N_{i}$ of the required form for $i>0$ and $N_{1}$ central in $N$.

Since $G$ has $\max -n, N_{1}$ is finitely generated as a normal subgroup of $G$-by $n_{1}, \ldots, n_{i}$, say. Also, since $C / N$ is abelian, we can choose $x_{1}, \ldots, x_{k} \in C$ such that each element $g$ of $C$ can be written in the form $g=n x_{1}^{\alpha(1)} \ldots x_{k}^{\alpha(k)}\left(n \in N, \alpha_{i} \in J\right)$. Thus; as $N_{1}$ is central in $N$,

$$
N_{1}=\left\langle n_{j}^{x_{1}^{\alpha(1)} \ldots x_{k}^{\alpha(k)} x^{\beta}}: \alpha(i), \beta \in J, j=1, \ldots, t\right\rangle .
$$

But, as $x_{1}$ has polycyclic action on $n_{j},\left\langle n_{j}^{x_{1}^{\alpha(1)}}: \alpha(1) \in J\right\rangle$ is finitely generated and so,

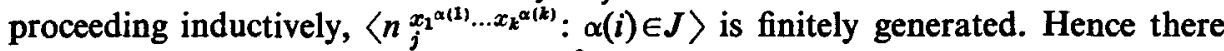
exist $d_{1}, \ldots, d_{s} \in N_{1}$ such that $N_{1}=\left\langle d_{j}^{x^{\beta}}: \beta \in J, j=1, \ldots, s\right\rangle$.

We can now refine the above series of $N$ by

$$
\{1\} \leqslant\left\langle d x_{1}^{x^{j}}: j \in J\right\rangle \leqslant \ldots \leqslant\left\langle d_{1}^{x^{j}}, \ldots, d_{s}^{x^{j}}: j \in J\right\rangle=N_{1} \leqslant \ldots
$$

which completes the proof of the lemma.

LEMMA 5. Let $K$ be an algebraic number field and let $K^{*}$ denote the multiplicative group of non-zero elements of $K$. Suppose that $G$ is a finitely generated subgroup of the $n$-fold direct power $K^{*} \times \ldots \times K^{*}$ of $K^{*}$ with the following property:

$$
\text { if }\left(\alpha_{1}, \ldots, \alpha_{n}\right) \in G \text {, then either all } \alpha_{i} \text { or all } \alpha_{i}^{-1} \text { are algebraic integers. }
$$

Then $G$ has a normal subgroup $N$ satisfying:

(a) $G / N$ is cyclic;

(b) if $\left(\alpha_{1}, \ldots, \alpha_{n}\right) \in N$, then each $\alpha_{i}$ is a unit in the ring of algebraic integers of $K$.

(For basic facts about algebraic number fields, see Artin, 1968.)

ProOF. If $k \in K^{*}$, let $\varphi(k)$ be the absolute value of the norm of $k$ in $K$. Let $\bar{\varphi}: K^{*} \times \ldots \times K^{*} \rightarrow Q^{*}$ (where $Q$ is the field of rationals) be given by

$$
\bar{\varphi}:\left(\alpha_{1}, \ldots, \alpha_{n}\right) \mapsto \varphi\left(\alpha_{1}\right) \ldots \varphi\left(\alpha_{n}\right)
$$

and denote $G \bar{\varphi}$ by $\boldsymbol{G}$. The condition given for $G$ implies that, for each $a \in \bar{G}, a$ is either a natural number or the inverse of a natural number. 
Choose $b$ to be the least natural number in $\bar{G}$ not equal to 1 and let $c \in G$. Then we can choose $\varepsilon= \pm 1$ so that $c^{\varepsilon}$ is a natural number and hence $b^{m} \leqslant c^{\varepsilon}<b^{m+1}$ for some natural number $m$. Thus $1 \leqslant b^{-m} c^{\epsilon}<b$ and so, $b^{-m} c^{\varepsilon}$ is a natural number in $\bar{G}$ which is less than $b$. Hence $b^{-m} c^{\varepsilon}=1$, that is, $c=b^{m \varepsilon}$ and so $c \in\langle b\rangle$. Thus $\bar{G}=\langle b\rangle$.

Let $N$ be the kernel of $\vec{\varphi}$. Then $G / N \cong G$ and so $G / N$ is cyclic. Also, if $\left(\alpha_{1}, \ldots, \alpha_{n}\right) \in N$, then $\varphi\left(\alpha_{1}\right) \ldots \varphi\left(\alpha_{n}\right)=1$ and so, because we have that either each $\varphi\left(\alpha_{i}\right)$ or each $\varphi\left(\alpha_{i}\right)^{-1}$ is a natural number, it follows that each $\varphi\left(\alpha_{i}\right)$ is 1 . Hence each $\alpha_{i}$ is a unit in the ring of algebraic integers of $K$, as required.

\section{Semi-polycyclic groups are $\mathfrak{T}$-groups}

We begin by showing that groups in $\mathfrak{I}$ are finitely presented and so, as $\mathfrak{Q I}=\mathfrak{I}$, have max- $n$. This will then be used to show that $\mathfrak{I}=\mathfrak{Q}_{1}$.

Proposition 6. Each group in $\mathfrak{T}$ is finitely presented.

Proof. Let $G \in \mathfrak{T}$ and let

$$
\{1\} \leqslant N \leqslant C \leqslant H \leqslant G
$$

be a $\mathfrak{T}$-series for $G$. Since finite extensions of finitely presented groups are finitely presented, it clearly suffices to suppose that $H=G$. Suppose also that $G=\langle C, x\rangle$. By Lemma 4, there exists a finitely generated subgroup $D$ of $N$ such that $N=\left\langle D^{x^{3}}: j \in J\right\rangle$. Since $C / N$ is finitely generated, it follows that there exists a finitely generated subgroup $D_{1}$ of $C$ such that $E=\left\langle D_{1}^{x^{3}}: j \in J_{+}\right\rangle$(say) is finitely generated. Let $\varphi: E \rightarrow E$ be the monomorphism of $E$ induced by conjugation by $x$. Then $G$ clearly has the presentation

$$
\left\langle x, E: E \varphi=E^{x}\right\rangle .
$$

Since $C$ is locally polycyclic, $E$ is polycyclic and so finitely presented. Thus $G$ is finitely presented.

The bulk of the proof in showing that semi-polycyclic groups are $\mathfrak{I}$-groups is to show that they have finite rank. We accomplish the major portion of this in the next lemma.

LemmA 7. Let $G \in Q_{1}$ and suppose that $G$ has an abelian normal subgroup $A$ which is either torsion-free or of finite prime exponent. If $G / A \in \mathfrak{T}$, then $A$ has finite rank. 
Proof. We give the proof only in the case that $A$ is torsion-free; the case when $A$ has finite exponent is similar.

Let

$$
A / A \leqslant N / A \leqslant C / A \leqslant H / A \leqslant G / A
$$

be a $I$-series of $G / A$. As usual, it suffices to assume that $G=H$. Because $A$ is torsion-free, it naturally embeds in the tensor product $A=A \otimes_{\mathrm{J}} Q$ (where $Q$ is the field of rationals) and $A$ has finite rank if and only if $\bar{A}$ does. Observe that $\bar{A}$ is naturally a $Q(G / A)$-module; we use additive notation for the multiplication in $\bar{A}$, so that, if $g \in G / A$ and $m \in \bar{A}, m \cdot g$ denotes $g^{-1} m g$ and $Q\langle\ldots\rangle$ denotes "the rational subspace of $\bar{A}$ generated by ...".

We commence the proof with some observations.

(1) If $m \in \bar{A}, g \in G / A$, then, because $g$ has semi-polycyclic action on $m$, $Q\left\langle m: g^{i}: i \in J\right\rangle$ has finite dimension.

(2) If $m \in \bar{A}$ and $N_{1}$ is a finitely generated subgroup of $N / A$, then $N_{1}$ has a generating set $g_{1}, \ldots, g_{k}$ so that each $g \in N_{1}$ can be written in the form $g_{1}^{\alpha_{1}} \ldots g_{k}^{\alpha_{k}}$ $\left(\alpha_{i} \in J\right)$ and so, using (1) ${ }_{Q}\left\langle m \cdot g: g \in N_{1}\right\rangle$ has finite dimension.

(3) If $m \in \bar{A}, d \in N / A$ and $g \in G / A$, then, as $g$ has semi-polycyclic action on $N / A$, we can suppose, without loss of generality, that $D_{+}=\left\langle\alpha^{g^{i}}: i \in J_{+}\right\rangle$is finitely generated. Hence, using comments (1) and (2)

$$
{ }_{Q}\left\langle m \cdot g^{i} e g^{j}: i, j \in J, e \in D_{+}\right\rangle
$$

has finite dimension. But, if $h \in D=\left\langle d^{g^{i}}: i \in J\right\rangle$, then $h^{g^{j}}=h_{1} \in D_{+}$for some $j$ and so

$$
{ }_{Q}\langle m \cdot h\rangle \leqslant{ }_{Q}\left\langle m \cdot g^{j} h_{1} g^{-j}\right\rangle \leqslant{ }_{Q}\left\langle m \cdot g^{i} e g^{j}: i, j \in J, e \in D_{+}\right\rangle .
$$

Hence ${ }_{Q}\langle m \cdot h: h \in D\rangle$ has finite dimension.

Suppose now that

$$
A / A \leqslant N_{1} / A \leqslant \ldots \leqslant N_{R} / A=N / A
$$

is the finite central series of $N / A$ guaranteed by Lemma 4 ( $G / A$ has max- $n$ by Proposition 6). Then a repeated application of (3) shows that, if $m \in M$,

$$
{ }_{Q}\langle m \cdot g: g \in N \mid A\rangle \text { has finite dimension. }
$$

Now $G / N$ is a finitely generated abelian group, and so, by repeated application of (1), combined with (4), if $m \in \bar{A}$

$$
Q^{\langle m \cdot g: g \in G / A\rangle} \text { has finite dimension. }
$$

Finally, $G / A \in \mathfrak{T}$ and so is finitely presented, by Proposition 6 , and so $A$ is a finitely generated $Q(G / A)$-module. Hence, by (5), $A$ has finite dimension, which completes the proof of the lemma. 
Proposition 8. Let $G$ be a finitely generated soluble group with an abelian normal subgroup $A$. Suppose that $G / A \in \mathfrak{I}$ and $G$ has semi-polycyclic action on $A$. Then $G \in \mathfrak{I}$.

Proof. Since, by Proposition 6, each group in $\mathfrak{I}$ is finitely related, $G$ has a quotient maximal with respect to not lying in $\mathfrak{I}$. Without loss of generality, we can assume $G$ is this quotient. Since, by Proposition 6 , each group in $\mathfrak{I}$ has $\max -n$, it follows that $G$ also has max- $n$.

Let $T$ be the torsion subgroup of $A$. Then $T$ is normal in $G$ and, since $G$ has $\max -n, T$ has finite exponent. By Lemma 7 , and the fact that every proper quotient of $G$ is in $\mathcal{I}$ it follows that $T$ is finite. But then, by Lemma 1 , it suffices to assume $T$ is trivial.

Hence $A$ is torsion-free, and so, by Lemma 7, of finite rank. Let $K$ be the centralizer of $A$ in $G$. Then $G / K$ can be regarded as a matrix group of finite degree over the rational numbers. Thus, by Mal'cev's theorem (see 3.6 of Wehrfritz, 1973), $G / K$ has a triangularizable normal subgroup $H / K$ of finite index in $G / K$.

Each element of $G$ has semi-polycyclic action on $A$ and so each matrix $g$ in $H / K$ satisfies an equation

$$
\alpha_{n} g^{n}+\ldots+\alpha_{0}=0
$$

with each $\alpha_{i}$ an integer and either $\alpha_{n}$ or $\alpha_{0}$ equal to 1 . Thus the eigenvalues of $g$ are either all algebraic integers or all inverses of algebraic inters. Since $H / K$ is triangularizable, Lemma 5 applies to show that there is a subgroup $C / K$ of $H / K$ (which we can assume normal in $G / K$ ) such that $H / C$ is cyclic and the eigenvalues of any matrix in $C / K$ are units in the ring of algebraic integers.

Let $N / K$ be the unitriangularisable subgroup of $H / K$. Observe that $N / K$ is unipotent; that is, if $a \in A, g \in N$, then $[a, n g]=1$ for some fixed natural number $n$.

We now have a normal series of $G$

$$
\{1\} \leqslant A \leqslant K \leqslant N \leqslant C \leqslant H \leqslant G .
$$

Let

$$
A / A \leqslant N_{1} / A \leqslant C_{1} / A \leqslant H_{1} / A \leqslant G / A
$$

be a $\mathfrak{T}$-series for $G / A$ and denote $H \cap H_{1}, C \cap H_{1}, N \cap N_{1}$ by $H_{2}, C_{2}, N_{2}$ respectively. We claim that

$$
\{1\} \leqslant N_{2} \leqslant C_{2} \leqslant H_{2} \leqslant G
$$

is a $\mathfrak{I}$-series for $G$. For,

(a) since $G / H$ and $G / H_{1}$ are both finite, so is $G / H_{2}$;

(b) $\mathrm{H}_{2} / \mathrm{C}_{2} \cong \mathrm{C}\left(\mathrm{H \cap} \mathrm{H}_{1}\right) / \mathrm{C} \leqslant \mathrm{H} / \mathrm{C}$ and so $\mathrm{H}_{2} / \mathrm{C}_{2}$ is cyclic;

(c) $H_{2}^{\prime} \leqslant H^{\prime} \cap H_{1}^{\prime} \leqslant N \cap N_{1}=N_{2}$ and so $H_{2} / N_{2}$ is abelian; 
(d) $N \cap N_{1} / A \leqslant N_{1} / A$ and so $N \cap N_{1} / A$ is nilpotent; also, as $N \cap N_{1} / A \leqslant N / A$, each element $g \in N \cap N_{1} / A$ satisfies $[A, n g]=1$ for some $n$ independent of $g$; thus $N \cap N_{1}$ is nilpotent;

(e) if $g \in C_{2}$, then $g K \in C / K$, and so $g$ has polycyclic action on $A$ ("the eigenvalues of $g K$ are units")-also $g A \in C_{1} A$ and so $g$ has polycyclic action on $N_{2} / A$; thus $g$ has polycyclic action on $N_{2}$.

Hence $G$ has a $\mathfrak{I}$-series and so $G \in \mathfrak{I}$-which completes the proof.

THEOREM 9. The class of semi-polycyclic groups is precisely the class of $\mathfrak{T}$-groups

Proof. The fact that $\mathfrak{I} \subseteq \mathfrak{Q}_{1}$ is Proposition 3 and the reverse implication follows directly from Proposition 8 using a straightforward induction on solubility length.

\section{Coherent groups are semi-polycyclic}

We begin by showing that finitely generated coherent soluble groups have $\max -\boldsymbol{n}$.

Proposition 10. A finitely generated coherent soluble group $G$ has max- $n$.

Proof. Let $l(G)$ denote the solubility length of $G$ and let $m(G)$ denote $\min \{$ torsion-free ranks of $G / H$ where $H \unlhd G, G / H$ is abelian

and $H$ is soluble of length $l(G)-1\}$.

The proof is by a double induction on $l(G)$ and $m(G)$.

Choose $H \unlhd G$ so that $G / H$ is abelian, $H$ has length $l(G)-1$ and the torsion-free rank of $G / H$ is $m(G)$. If $m(G)=0$, then $G$ is a finite extension of $H$ and, as $H$ has $\max -n$ by induction on $l(G), G$ thus has $\max -n$.

Suppose then that $m(G)>0$ and choose $N$ so that $H \leqslant N \unlhd G$ and $G / N$ is infinite cyclic. Let $G=\langle N, t\rangle$ for $t \in G$. Now $G$ is finitely presented and so by Theorem A of Bieri and Strebel (1977) it is an ascending HNN-extension with finitely generated base group $B \leqslant N$ and stable letter $t$. Induction on $m(G)$ now shows that $B$ has $\max -n$.

Let $M \unlhd G$. It suffices to show that $M$ is finitely generated as a normal subgroup of $G$ and so, as $M / M \cap N=M N / N$ is cyclic, that $M \cap N$ is finitely generated as normal subgroup; that is, we can suppose $M \leqslant N$. Now $M \cap B$ is normal in $B$ and so, as $B$ has $\max -n, M \cap B=\left\langle m_{1}, \ldots, m_{k}\right\rangle^{B}$ for some $m_{1}, \ldots, m_{k} \in M \cap B$. But if $m \in M$, then $m^{m} \in M \cap B$ for a suitably chosen positive power $t^{n}$ of $t$. Thus $m^{m} \in\left\langle m_{1}, \ldots, m_{k}\right\rangle^{B}$ and so $m \in\left\langle m_{1}, \ldots, m_{k}\right\rangle^{G}$. Hence $M=\left\langle m_{1}, \ldots, m_{k}\right\rangle^{G-\text { which }}$ completes the proof. 
Recall that the groups $M(\alpha, p)$ are defined as matrix groups, over a field of characteristic $p$, generated by

$$
\left\{\left[\begin{array}{ll}
1 & 0 \\
1 & 1
\end{array}\right]\left[\begin{array}{ll}
\alpha & 0 \\
0 & 1
\end{array}\right]\right\} .
$$

The following is a simple application of Theorem C of Bieri and Strebel (1977).

LEMMA 11. The group $M(\alpha, p)$ is finitely presented if and only if

(i) $p \neq 0$ and $\alpha$ is algebraic over the prime field, or

(ii) $p=0$ and at least one of $\alpha$ or $\alpha^{-1}$ is an algebraic integer.

In order to investigate necessary conditions for coherence, we introduce an expanded version of the class $\mathfrak{Q}_{1}$ of semi-polycyclic groups.

The class of finitely generated soluble groups $G$ satisfying:

for all $x, y \in G$ with $\left\langle x^{v^{i}}: i \in J\right\rangle$ abelian,

either $\left\langle x^{y^{i}}: i \in J_{+}\right\rangle$or $\left\langle x^{y^{i}}: i \in J_{-}\right\rangle$is finitely generated,

will be denoted by $\mathfrak{Q}_{2}$.

It is easily seen that a group $M(\alpha, p)$ belongs to $\mathfrak{Q}_{2}$ precisely if it is finitely presented. Extending this gives a necessary condition for coherence.

LEMMA 12. Let $G$ be a finitely generated soluble group and suppose $G \notin Q_{2}$. Then $G$ contains a subgroup isomorphic to $M(\alpha, p)$ with $p \neq 0$ and $\alpha$ transcendental over the prime field or $p=0$ and neither $\alpha$ nor $\alpha^{-1}$ an algebraic integer. In particular, $G$ is not coherent.

Proof. Since $G \notin Q_{2}$, there exist elements $x, y \in G$ such that $A=\left\langle x^{y^{3}}: i \in J\right\rangle$ is abelian and neither $\left\langle x^{v^{i}}: i \in J_{+}\right\rangle$nor $\left\langle x^{v^{i}}: i \in J_{-}\right\rangle$is finitely generated. Without loss of generality, we can assume $G=\langle x, y\rangle$.

Let $T$ be the torsion subgroup of $A$. Since $G$ is metabelian and so has max-n, $T$ has finite exponent. Thus if $T$ is infinite it contains an infinite subgroup $S$, normal in $G$, of prime exponent $p$. If $\left\langle s^{x^{i}}: i \in J\right\rangle$ is infinite for some $s \in S$, then $\langle s, x\rangle \cong M(\alpha, p)$ with $\alpha$ transcendental. So we can suppose $\left\langle s^{x^{i}}: i \in J\right\rangle$ is finite for each $s \in S$. But then, as $G$ has $\max -n$ and so $S$ is the normal closure of a finite number of elements, $S$ is finite. Thus we can suppose $T$ is finite.

Since $G$ is metabelian, $G$ is residually finite and so there exists a subgroup $H$ of finite index in $G$ such that $H \cap T=\{1\}$. Clearly $H \cap A$ must contain an element $w$ such that neither $\left\langle w^{v^{i}}: i \in J_{+}\right\rangle$nor $\left\langle w^{\nu^{i}}: i \in J_{-}\right\rangle$is finitely generated and so it suffices to assume that $H=G$ or, equivalently, that $A$ is torsion-free. But then 
$G \cong M(\alpha, 0)$ for some $\alpha$ and so, as $G \notin \mathfrak{Q}_{2}$, neither $\alpha$ nor $\alpha^{-1}$ can be an algebraic integer. Lemma 10 again shows that $G$ is therefore not coherent.

Unfortunately, perhaps, the classes $\mathfrak{Q}_{1}$ and $\mathfrak{Q}_{2}$ are distinct; for example, straightforward calculations show that the two generator relatively free centre-bymetabelian groups lies in $\mathfrak{Q}_{2}$ but not in $\mathfrak{Q}_{1}$. (It can be shown, although we do not offer a proof here, that the classes coincide for abelian by nilpotent groups.) There is, however, a strong relationship between them.

LEMMA 13. If $G$ is a finitely generated soluble group and every quotient of $G$ lies in $\mathfrak{Q}_{2}$, then $G \in \mathfrak{Q}_{1}$.

Proof. By induction on the solubility length of $G$. Let $A$ be the last non-trivial term of the derived series of $G$. By the inductive hypothesis, $G / A \in Q_{1}$ and so, by Theorem 9, $G / A \in \mathfrak{T}$. Since every element of $G$ has polycyclic action on $A$ (because $G \in \mathfrak{Q}_{2}$ ), Proposition 8 implies that $G \in \mathfrak{I}$. Hence $G \in \mathfrak{Q}_{1}$, as required.

COROLLARY 14. If a finitely generated soluble group $G \notin \mathbb{Q}_{1}$, then some subgroup of a quotient group of $G$ is isomorphic to an infinitely presented $M(\alpha, p)$.

The proof that finitely generated soluble coherent groups are semi-polycyclic is now obtained by a simple combination of Proposition 10 and Corollary 14.

\section{References}

Emil Artin (1968), Algebraic Numbers and Algebraic Functions (Nelson, London).

Gilbert Baumslag (1973), "Subgroups of finitely presented metabelian groups", J. Austral. Math. Soc. 16, 98-110.

Gilbert Baumslag (1974), "Finitely presented metabelian groups", Proc. Second Internat. Conf. Theory of Groups, Canberra, 1973, pp. 65-74 (Lecture Notes in Mathematics 372, Springer-Verlag, Berlin, Heidelberg, New York).

Robert Bieri and Ralph Strebel (to appear), "Almost finitely presented soluble groups".

Derek J. S. Robinson (1972), Finiteness Conditions and Generalized Soluble Groups, Parts 1 and 2 (Ergebnisse der Mathematik und ihrer Grenzgebiete, Band 62, 63. Springer-Verlag, Berlin, Heidelberg, New York, 1972).

B. A. F. Wehrfritz (1973), Infinite Linear Groups (Ergebnisse der Mathematik und ihrer Grenzgebiete, Band 76. Springer-Verlag, Berlin, Heidelberg, New York).

University of Melbourne

Parkville, Victoria 3052

Australia 